\title{
A Ferrite LTCC-Based Monolithic SIW Phased Antenna Array
}

\author{
Ahmed Nafe, Student Member, IEEE, Farhan A. Ghaffar Student Member, IEEE, \\ M. F. Farooqui, Student Member, IEEE, and Atif Shamim, Senior Member, IEEE
}

\begin{abstract}
In this work, we present a novel configuration for realizing monolithic SIW-based phased antenna arrays using Ferrite LTCC technology. Unlike the current common schemes for realizing SIW phased arrays that rely on surface-mount component ( $p-i-n$ diodes, etc) for controlling the phase of the individual antenna elements, here the phase is tuned by biasing of the ferrite filling of the SIW. This approach eliminates the need for mounting of any additional RF components and enables seamless monolithic integration of phase shifters and antennas in SIW technology. As a proof of concept, a two-element slotted SIW-based phased array is designed, fabricated and measured. The prototype exhibits a gain of $4.9 \mathrm{dBi}$ at $13.2 \mathrm{GHz}$ and a maximum E-plane beam-scanning of \pm 28 degrees using external windings for biasing the phase shifters. Moreover, the array can achieve a maximum beam-scanning of \pm 19 degrees when biased with small windings that are embedded in the package. This demonstration marks the first time a fully monolithic SIW-based phased array is realized in Ferrite LTCC technology and paves the way for future larger-size implementations.
\end{abstract}

Index Terms-Ferrite phase shifter, low-temperature co-fired ceramic (LTCC), phased array, substrate integrated waveguide (SIW).

\section{INTRODUCTION}

Antennas having beam-scanning functionality are required in radars and many modern communication and imaging systems. Over the years, many different implementations of beamscanning antenna arrays have been carried out in different technologies (waveguides, microstrip, etc). In the past decade, Substrate Integrated Waveguide (SIW) concept has become a popular platform for realizing antennas and circuits [1]. SIWs combine the simplicity and low-loss nature of rectangular waveguides while being low-cost and easy to fabricate and integrate with planar circuitry.

Most of the developed SIW-based beam-scanning arrays in literature rely on a fixed beam-forming network such as a butler matrix or a rotman lens [2], [3]. The disadvantage of such approach is that the beam can only be switched over a discrete set of scan angles and that the size of the beam-forming network grows quickly with the number of required beam directions [4], [5]. The need of such complex networks can be eliminated by using tunable phase shifters and implementing a phased array with continuous beam-steering capability.

A problem in tunable phase shifters is that their integration with the SIW typically requires surface-mount components.

The authors are with the Department of Electrical Engineering, King Abdullah University of Science and Technology (KAUST), Kingdom of Saudi Arabia, Thuwal, Jeddah 23955-6900, e-mail: ahmed.nafe@kaust.edu.sa

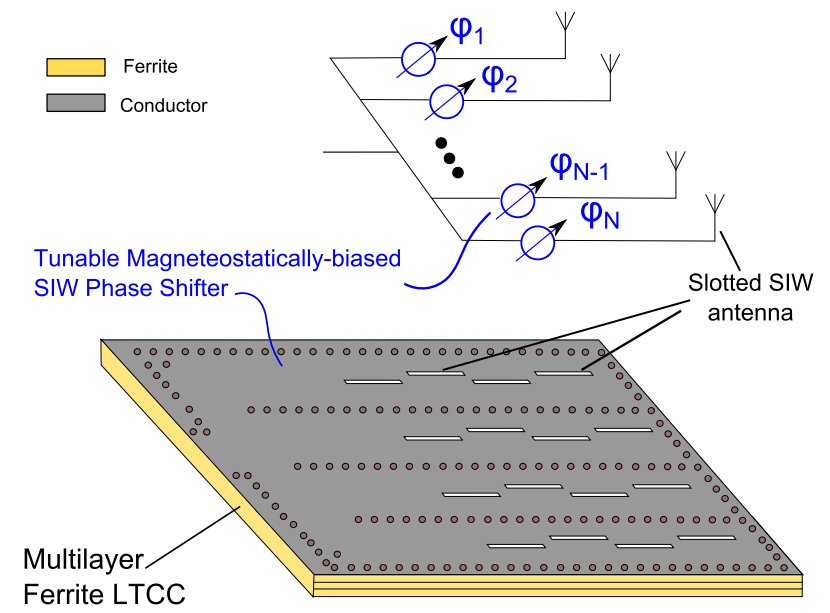

Fig. 1: Conceptual sketch of a monolithic slotted SIW phased antenna array in Ferrite LTCC.

For example, the phase shifter implemented in [6] required using four $\mathrm{p}-\mathrm{i}-\mathrm{n}$ diodes as switches which are mounted on the SIW. Mounting tunable components such as varactor diodes or switches on the SIW increases the phase shifter complexity and adds parasitics which may become significant at high frequencies. In this work, we avoid the above-mentioned issues by using a controllable medium (ferrite) as a multilayer substrate for the SIW.

Generally, conventional ferrite materials have been extensively used for implementing tunable phase shifters in planar transmission-line (microstrip, CPW, etc) [7]-[10] as well as non-planar waveguide configurations [11]. But even though it has been over a decade since the invention of SIWs, only very few ferrite-based SIW phase shifters have been reported [12], [13] and these have not been demonstrated in any kind of phased array system. A possible reason for the limited demonstrations is the difficulty of integration of SIW and conventional ferrites. The brittle ceramic nature of ferrite substrates makes the realization of via rows without substrate fracture a real challenge. To circumvent the problem, researchers in [13], [14] used ferrite slabs to load a SIW that is implemented in a conventional duroid substrate. Although this approach might be acceptable if few devices are needed, it is not feasible for implementing medium and large phased antenna arrays due the need for extensive machining for each device.

A more elegant way to resolve ferrite-SIW integration problems is through the use of new technology called 'Ferrite 


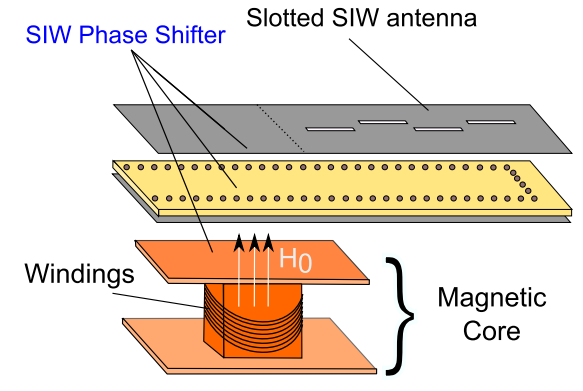

(a)

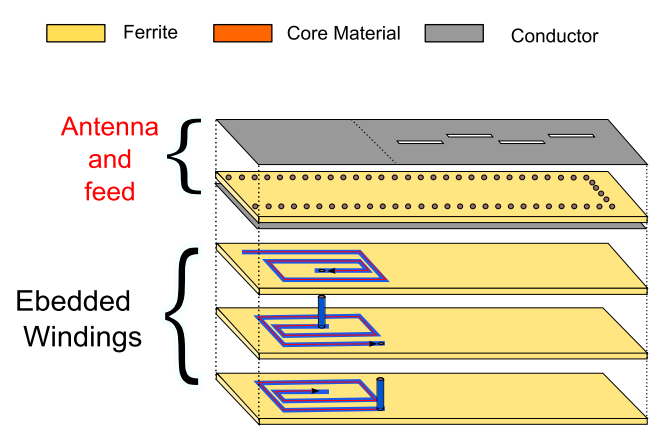

(b)

Fig. 2: Conceptual sketch of (a) tunable SIW phase shifter biased using external circuitry and (b) tunable SIW phase shifter biased using embedded circuitry within the Ferrite LTCC package.

LTCC' [15]-[19]. In Ferrite LTCC, the traditional dielectric layers of conventional LTCC are replaced by ferrite ones. The conductor traces and vias are implemented in the unfired tape which is much easier to process. This makes it possible to have a multilayer ferrite package with printed conductors and vias and hence allows the realization of SIWs.

Using Ferrite LTCC, monolithically integrated slotted-SIW phased antenna arrays can be realized as demonstrated in Fig. 1. An SIW subjected to a variable static magnetic bias field serves as a tunable phase shifter for each slotted SIW antenna arm. This bias field could be supplied by circuitry external to the package in the form of a winding around a magnetic core (as in Fig. 2(a)). Alternatively, by adding more layers, bias circuitry can be embedded within the ferrite package as shown in Fig. 2(b). The embedded windings approach provides the highest level of integration, however the number of turns of the windings which is proportional to the generated bias magnetic field is generally limited by the number of available LTCC layers. On the other hand, external biasing coils, though they occupy more space, are generally not restricted to the LTCC stack size and therefore can be made with large number of turns.

In this work, as a demonstration, we implemented a $2 \times 3 \mathrm{Ku}-$ band slotted-SIW antenna array with each $1 \times 3$ subarray being fed by a tunable phase shifter. Each phase shifter consists of a SIW that is subjected to a controllable anti-symmetric biasing magnetic field whose strength determines the insertion phase of the SIW. Two biasing schemes have been realized to tune the phase shifters. The first employs a coil system external to the LTCC package while the second relies on windings embedded within the Ferrite LTCC package. The array operation has been experimentally verified using both biasing techniques showing a beam scanning range of $\pm 28^{\circ}$ in the case of external windings and about $\pm 19^{\circ}$ in the case of embedded ones. The obtained results are encouraging and suggest the feasibility of this configuration for implementing Ku-band phased arrays for applications such as satellite communication systems.

\section{Design And Simulations}

A 10-layer LTCC stack is used for realizing the antenna array. The stack is made of ESL-40012 ferrite tape system [20]. This tape has a $\epsilon_{r} \approx 14.6, \tan \delta \approx 5 \times 10^{-3}$ and a

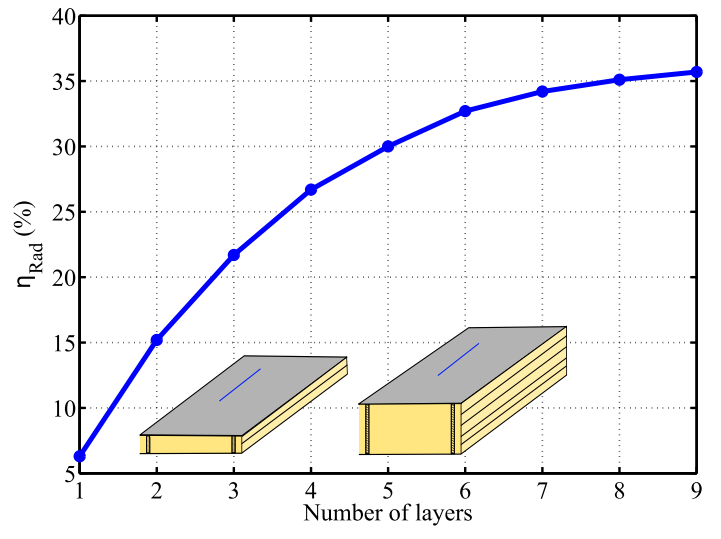

Fig. 3: Simulated radiation efficiency of a single slot versus number of SIW layers (each is $110 \mu \mathrm{m}$ thick ). The ferrite material is simulated in demagnetized state $\left(M=0, \mu_{d} \approx\right.$ 0.68 at $13 \mathrm{GHz}$ ) and silver is used for conductive traces and vias.

saturation magnetization $\left(4 \pi M_{s}\right)$ of 4000 Gauss that results in a magnetization frequency $\left(f_{m}\right)$ of about $11.2 \mathrm{GHz}$ [17]. The material is operated in the partially magnetized state $\left(0 \leq M \leq M_{s}\right)$ to minimize the bias field required. A design frequency of $13 \mathrm{GHz}$ is chosen to avoid the low-field losses that occur in such state at low frequencies and up to the material's magnetization frequency.

\section{A. Layer Assignment}

Since the 10-layer LTCC stack shall contain the antenna as well as the biasing circuitry, there will be a trade-off between the antenna performance (in RF) and dc biasing efficiency. Dedicating more layers for biasing circuitry would imply a denser winding with higher number of turns $(\mathrm{N})$ that will decrease the current needed to produce a given required bias magnetic field $(H \propto N I)$. However, dedicating more layers for the windings means less layers for the antenna which is expected to degrade the antenna performance (efficiency, gain, bandwidth). To examine how much the antenna efficiency is affected by the waveguide height, we simulated a single slot radiator (structure geometry similar to the model in Fig. 5 over different number of layers. The results, illustrated in 
Fig. 3, reveals that the radiation efficiency of the slot antenna increases as the number of layers is increased and it reaches a maximum value of about $36 \%$, relatively low due to the high ferrite permittivity, when all layers are dedicated for the antenna. It can also be noticed that the efficiency deteriorates quickly if less than 5 layers are used. Based on these results, it has been decided to dedicate the top five layers for the SIW and place the biasing circuitry in the bottom five layers.

\section{B. SIW Phase Shifter}

As mentioned earlier, the feeding SIW for each of the sub-arrays shall act as a phase shifter. The phase of the signal reaching each sub-array is controlled by biasing its feeding SIW-section. Biasing the SIW by a static magnetic field $H_{0}$ generates a magnetization level $M$ inside the material that dictates the effective permeability sensed by the RFsignal propagating through. For a partially magnetized ferrite biased in the y-direction, the permeability is described by the following tensor [15], [21]:

$$
[\mu]_{P M S}^{y-b i a s}=\mu_{0}\left[\begin{array}{ccc}
\mu & 0 & -j \kappa \\
0 & \hat{\mu} & 0 \\
j \kappa & 0 & \mu
\end{array}\right]
$$

Where the tensor elements are all functions of the magnetization level $M$ established in the material and the signal frequency $f$ as given by the equations below [22]:

$$
\begin{gathered}
\mu=\mu_{d}+\left(1-\mu_{d}\right)\left(\frac{M}{M_{s}}\right)^{\frac{3}{2}} \\
\hat{\mu}=\mu_{d}\left(1-\left(\frac{M}{M_{s}}\right)^{\frac{5}{2}}\right) \\
\kappa=n_{k} \frac{f_{m}}{f} \frac{M}{M_{s}}
\end{gathered}
$$

In the equations above, $\mu_{d}$ is the material's permeability when completely demagnetized $(M=0)$ and its value is given by the expression in (5). For the off-diagonal component $\kappa, n_{k}$ is a proportionality factor that is taken to be 0.5 at $13 \mathrm{GHz}$ for the material under consideration based on the previous works of [15].

$$
\mu_{d}=\frac{1}{3}+\frac{2}{3} \sqrt{1-\left(\frac{f_{m}}{f}\right)^{2}}
$$

Here, the phase shifting is achieved by applying an antisymmetric bias (depicted in Fig. 4) to each sub-array feeder. This anti-symmetric configuration of biasing has been studied in [23] and theoretically shown to induce large phase shift per unit length and to achieve very high figure of merit (phase shift per unit loss). In our recent work [19], we demonstrated a phase shifter with a figure of merit of about 100 degree/dB at Ku-band using this anti-symmetric configuration and biasing the device through 8 layers of embedded windings. In this work, we implement the phased array using a phase shifter of the same concept as in [19] but with slight modification in the layer assignment and biasing circuitry.

For the present case, to estimate the attainable phase shift, simulations were carried out for the 5-layer SIW being biased anti-symmetrically to different magnetization levels. In our

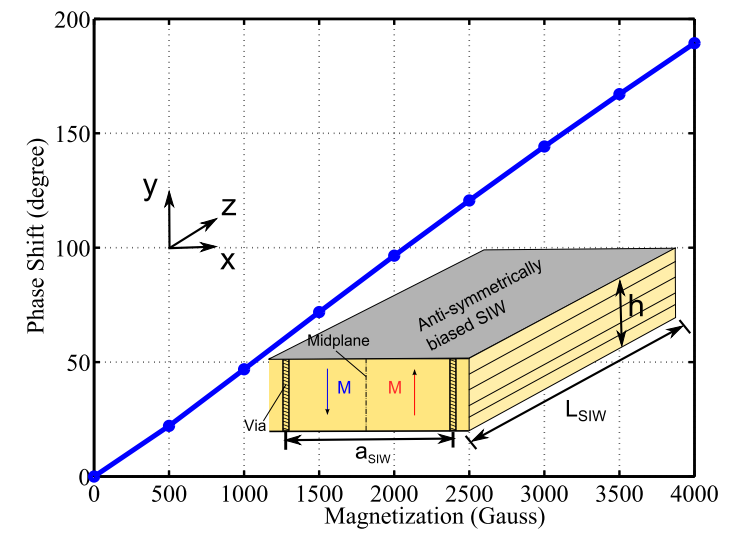

Fig. 4: Simulated phase shift versus magnetization level of an anti-symmetrically biased SIW $\left(a_{S I W}=4.5 \mathrm{~mm}, h=550\right.$ $\left.\mu \mathrm{m}, L_{S I W}=8 \mathrm{~mm}\right)$ at $13 \mathrm{GHz}$.

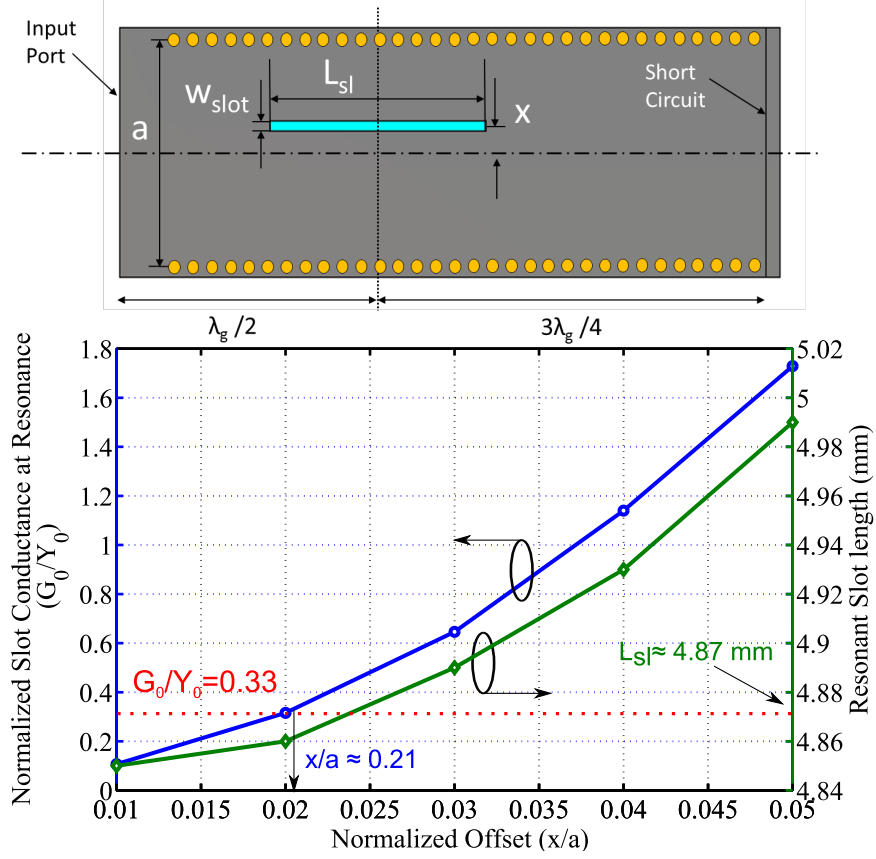

Fig. 5: Simulated slot conductance at resonance and resonant length for different slot offsets at $13 \mathrm{GHz}$ for five layers for a SIW width $\left(a=4.8 \mathrm{~mm}, w_{\text {slot }}=200 \mu \mathrm{m}\right)$.

simulation model, the partially magnetized material permeability definition is implemented using the aforementioned equations in CST microwave studio. The simulation results for an $8 \mathrm{~mm}$-long SIW feed section show that the forward insertion phase shift $\left(\angle S_{21}(M)-\angle S_{21}(M=0)\right)$ can reach about 180 degrees at $13 \mathrm{GHz}$ when the material is magnetized to saturation as illustrated in Fig. 4.

\section{Integrated Slotted SIW Phased Array}

To design a slotted antenna sub-array, first the characteristics of a single slot need to be obtained. A longitudinal slot on the broad face of rectangular waveguide can be modeled by a shunt load [24]. The slot length $L_{s l}$ will primarily determine the resonant frequency. While the slot offset from the SIW 

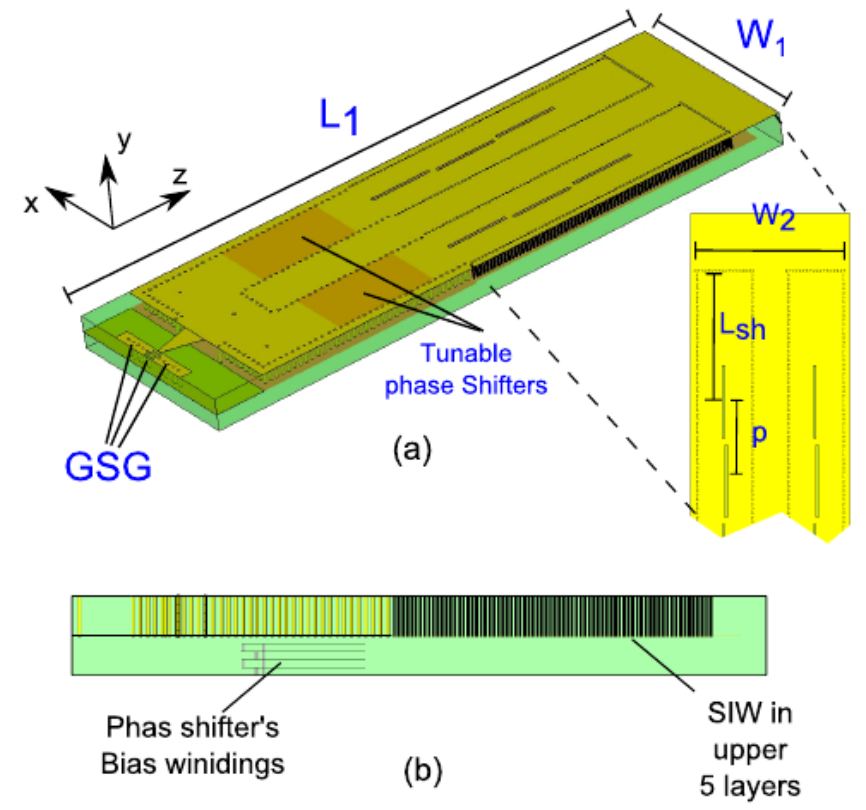

Fig. 6: Optimized 2x3 slotted-SIW array. (a) 3D view. (b) side view. All slots are $230 \mu \mathrm{m}$ wide, the rest of the dimensions are : $L_{1}=48 \mathrm{~mm}, W_{1}=14 \mathrm{~mm}, L_{s h} \approx 3 \lambda_{g} / 4=8.76 \mathrm{~mm}, L_{s l}=4.87$ $\left.\mathrm{mm}, p \approx \lambda_{g} / 2=5.3 \mathrm{~mm}, W_{2}=12.5 \mathrm{~mm}\right)$.

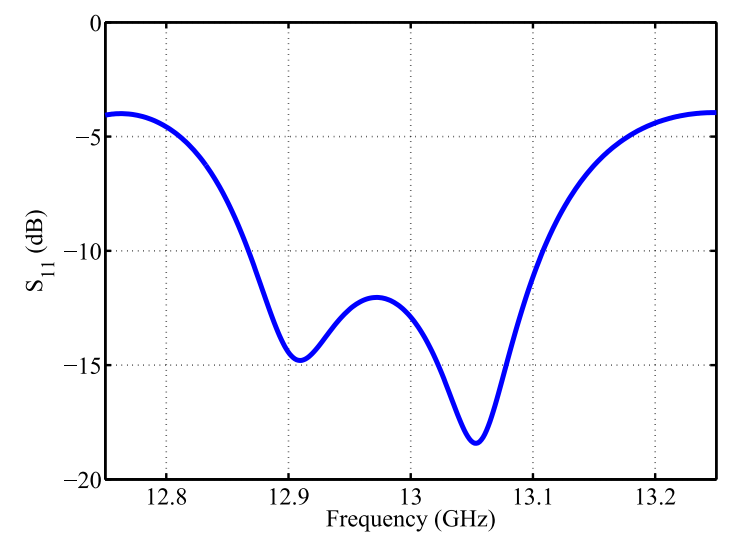

Fig. 7: Optimized $2 \times 3$ slotted-SIW array simulated reflection coefficient.

center (referred to using the symbol $x$ ) mainly dictates the slot conductance at resonance $G_{0}$. To extract the slot admittance, the procedure in [25] has been utilized. A single slot is simulated with the wave guide being terminated by a short circuit at a distance of $3 \lambda_{g} / 4$ form the slot center and with the port plane being at $\lambda_{g} / 2$ before the slot center. In this manner the input admittance at the port is equal to the admittance of the slot $Y_{\text {in }}=Y_{\text {slot }}$. Using this model, the slot length was varied at different offsets and each time the slot length that induces resonance at $13 \mathrm{GHz}$ and slot resonant conductance ( normalized to the waveguide characteristic admittance) $G_{0} / Y_{0}$ are recorded. The results for the slot conductance at resonance and slot resonant length at $13 \mathrm{GHz}$ are depicted in Fig. 5. The available LTCC panel space $(48 \times 14 \mathrm{~mm})$ could accommodate a maximum of three slots in addition to the

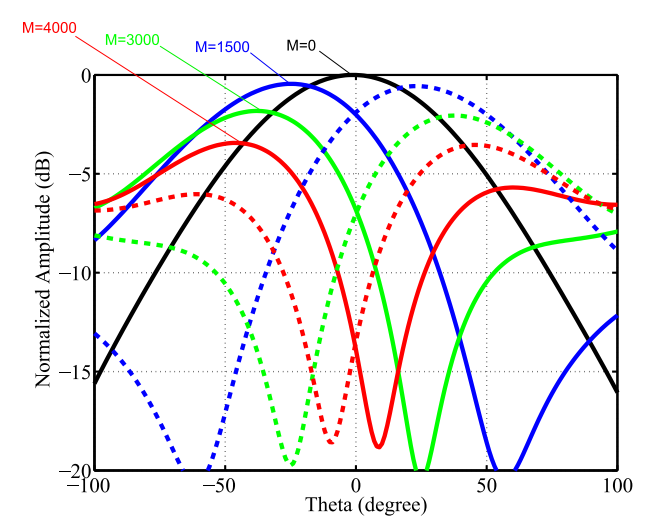

(a)

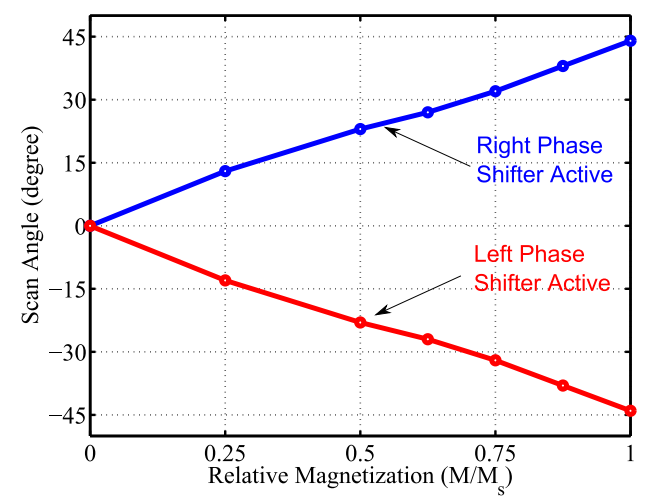

(b)

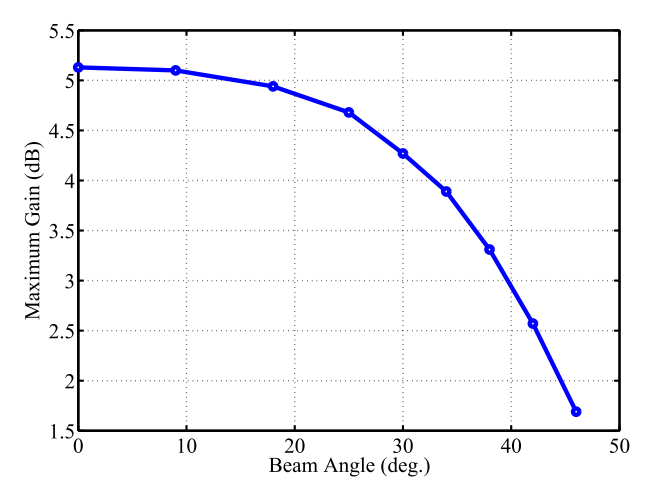

(c)

Fig. 8: Simulated beam steering. (a) E- plane radiation pattern (b) Beam angle versus magnetization (c) Gain over scan angle.

feeding structures. For a three-shunt-slots design, each slot should have resonant conductance equal to one-third of SIW's characteristic admittance $\left(\frac{G_{0}}{Y_{0}}=0.33\right)$. Using the shown characteristics curves of a single slot in Fig. 5, the resonantlength and offset were determined to be $4.87 \mathrm{~mm}$ and $100 \mu \mathrm{m}$ respectively.

Following this step, two three-slot sub-arrays were integrated with a phase shift section that is a simple extension of their feeding SIW. It is worth mentioning that a slightly larger SIW width is used for the antenna sub-array $(a=4.8$ $\mathrm{mm})$ than SIW for the phase shifter $(a=4.5 \mathrm{~mm})$. This small change in width has minor effects on the propagation and it helps relax the dimensions of the slot offset $(x \approx 0.21 a)$. 


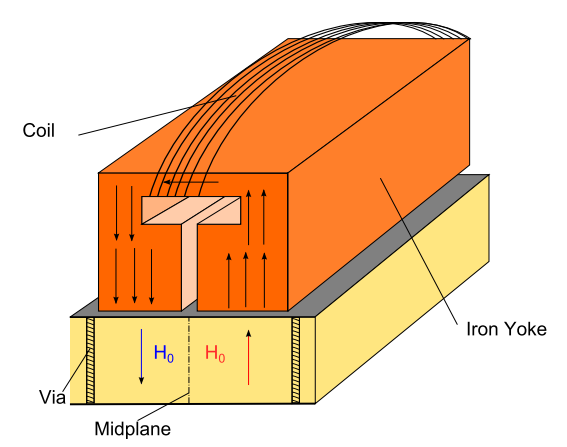

Fig. 9: Anti-symmetric biasing of a SIW using external coils and yokes.

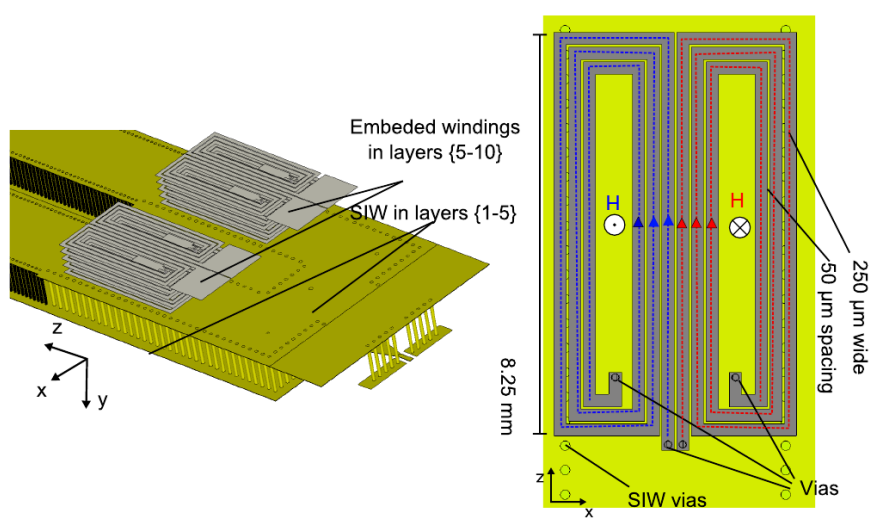

Fig. 10: Embedded windings for biasing individual phase shifters.

To feed the sub-arrays, a tapered microstrip to SIW transition has been designed and a 2-way SIW power divider that uses inductive vias for matching the impedance has been employed as depicted in Fig. 6. The whole structure has been optimized in CST microwave studio for operation at $13 \mathrm{GHz}$. Slight adjustments were required for the slot antenna dimensions obtained previously to account for mutual coupling effects. The simulated reflection coefficient for the optimized structure is illustrated in Fig. 7 showing that the array is matched better than $-12 \mathrm{~dB}$ at $13 \mathrm{GHz}$ and with a $10 \mathrm{~dB}$ impedance bandwidth extending from 12.88 to $13.1 \mathrm{GHz}$. The array exhibits a simulated gain of $5.1 \mathrm{dBi}$ and an efficiency of $41 \%$. As mentioned earlier, the relatively low efficiency and gain are due to the high permittivity of the ferrite substrate. However, it is expected that gain and efficiency numbers can be increased if more complex fabrication is used. For instance, creating an air cavity or perforating the substrate inside the SIW's antenna array portion is expected to reduce the effective permittivity and enhance radiation.

In this configuration, the antenna array beam scans in the Eplane towards positive and negative elevation angles depending on which phase shifter is being activated. A beam scanning range of \pm 45 degrees has been demonstrated in simulations by driving each phase shifter from the unbiased state $(M=0)$ to the saturated state $\left(M=M_{s}\right)$ as depicted in Fig. 8(a,b). The antenna remains matched when either phase shifter is biased with very little variation in $S_{11}$. As expected the gain drops

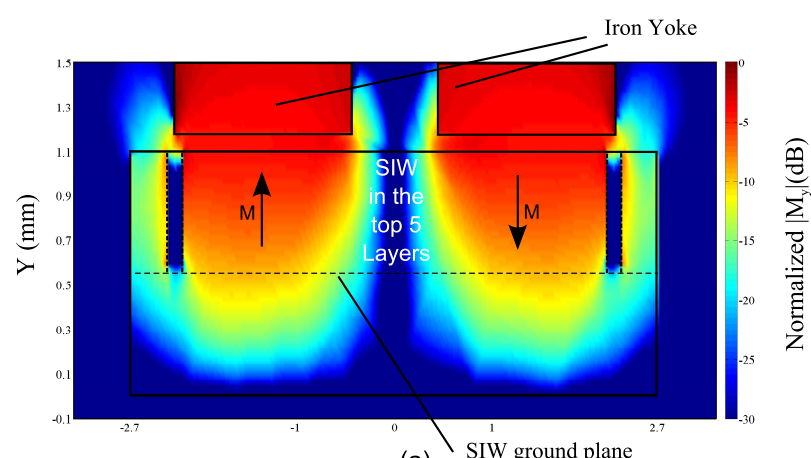

(a) SIW ground plane

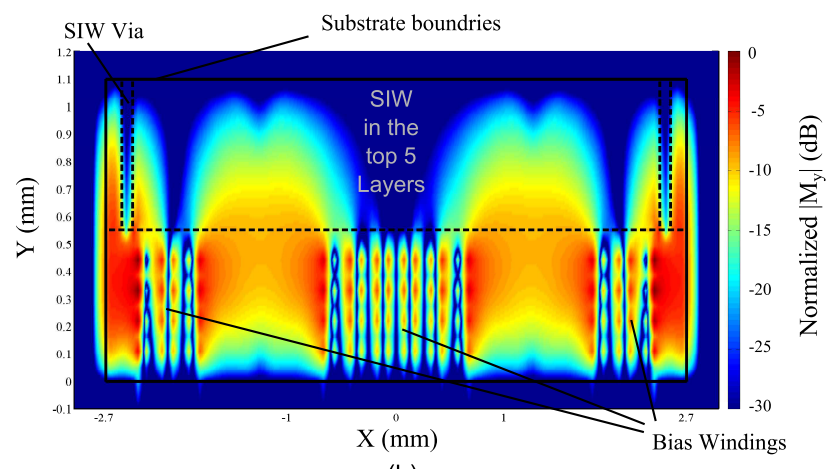

(b)

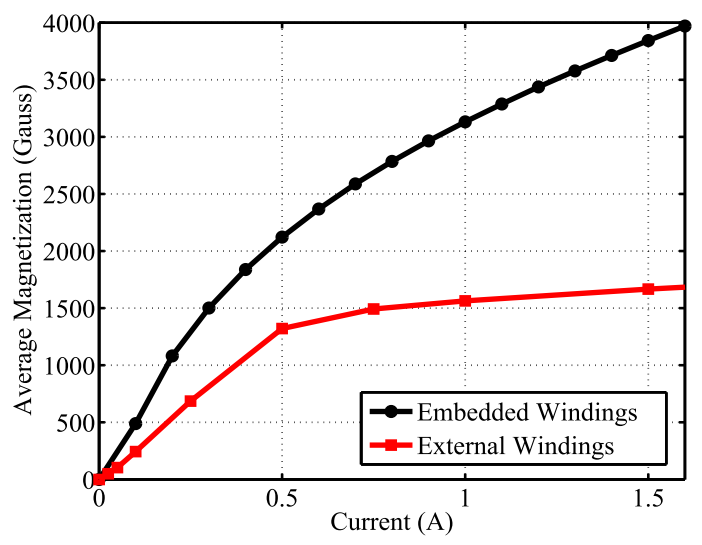

(c)

Fig. 11: Magnetostatic simulation results. Normalized Magnetization distribution $\left|M_{y}(x, y) / \max \left[M_{y}(x, y)\right]\right|$ over SIW cross section at bias current of 1 ampere. (a) external biasing , (b) embedded biasing and (c) average magnetization versus bias current.

when the array is steered from broadside. Steering the beam up to \pm 30 degrees is accompanied by slow drop in gain but after \pm 30 degrees the gain drops more quickly with the scan angle as seen in Fig. 8(c).

\section{Biasing Circuitry}

In order to magnetize the individual phase shifters, a magnetic field having an anti-symmetric configuration needs to be generated. In this work, we adopted two different approaches to generate such a configuration. In the first approach, an external coil system is used to bias the phase shifters. An 80-turn coil is wound around high permeability U-shaped iron 


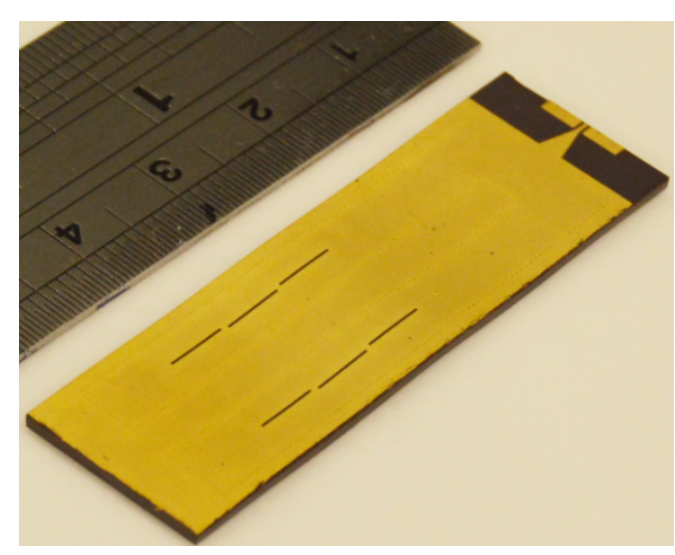

Fig. 12: Fabricated prototype.

yokes which are fitted above each phase shifter. This configuration helps guide the flux in anti-symmetric fashion inside the guide as demonstrated in Fig. 9 The second approach is by using windings embedded in the Ferrite LTCC package. The windings consist of 2 solenoid-like coils occupying the five layers beneath the SIW. The solenoids are symmetricallyplaced with respect to the SIW mid-plane with the current in the solenoids circulating in opposite sense to generate the required anti-symmetric bias magnetic field profile as illustrated in Fig. 10. Each solenoid has 3 turns in each layer, the largest turn has a length of $8.25 \mathrm{~mm}$ and a width of 2.7 $\mathrm{mm}$. Windings' dc pads are placed in the bottommost layer for dc supply connection. It is worth noting that here the $\mathrm{dc}$ windings are isolated from the microwave signal path by the SIW ground plane in layer five therefore there is no need for adding microwave chokes or dc block. The bias circuits are simulated separately using a magnetostatic solver (CST EM studio). Simulation results are depicted in Fig. 11.

As can be seen in Fig. 11(a,b), in both scenarios the magnetization is anti-symmetric (same in each half of the SIW but with opposite direction). However, the magnetization is not uniform within each half of the SIW. The average magnetization level over the SIW volume is calculated for each current excitation and the results are depicted in Fig. 11(c). As it is clear form the figure, in spite of having less total number of turns, the embedded windings can magnetize the structure to higher level with the same amount of dc current compared to the external biasing method. This is due to the demagnetization effects that occur due to the air gap existing between the external yoke and the ferrite substrate (assumed to be $100 \mu \mathrm{m}$ ). It is also worth mentioning that the bias magnetization fields are highly localized around their specific phase shifter. For the present phase shifter separation of $3.5 \mathrm{~mm}$, magnetizing one phase shifter to saturation (4000 Gauss) will magnetize the neighboring phase shifter to 4.4 Gauss $(0.11 \%)$. This would result in a very small induced phase shift of about 0.2 degrees in neighboring phase shifter.

\section{PRototype meAsurements Results AND DISCUSSION}

An array prototype, shown in Fig. 12, has been fabricated at VTT-electronics Finland. The prototype has been prepared

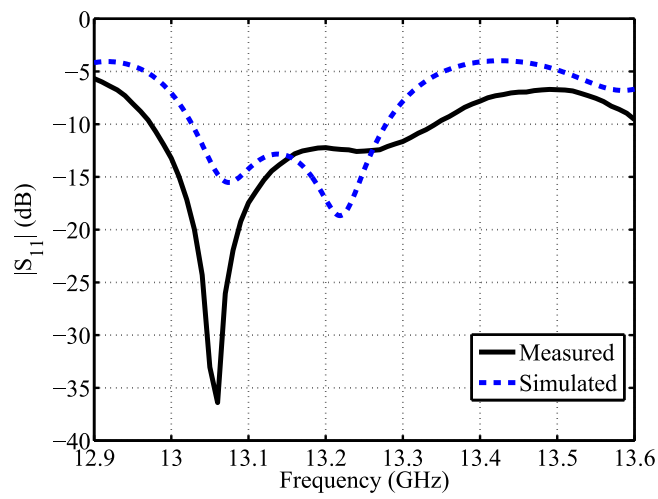

Fig. 13: Measured and post-measurement simulation of the array reflection coefficient.
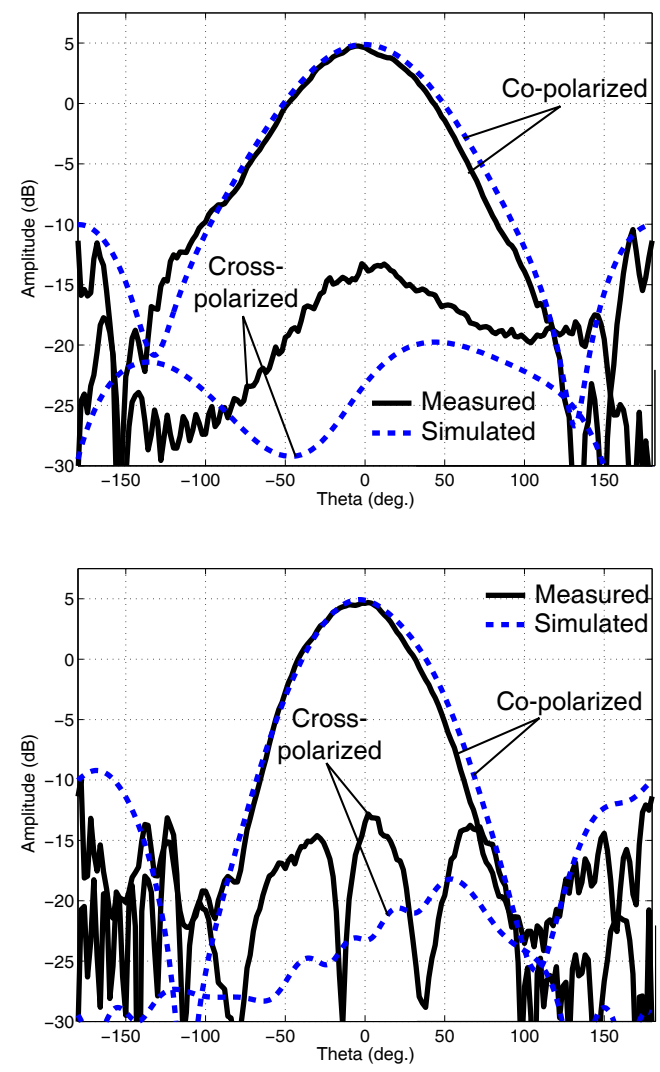

Fig. 14: Simulated and measured E (xy) plane (up) and H (yz) plane (down) at $13.2 \mathrm{GHz}$.

by firing at a peak temperature of 875 degree Celsius for 30 minutes.

\section{A. Measurements Results in Unbiased State}

An SMA connector has been mounted on the antenna and the input reflection coefficient has been measured using Agilent PNA-L 2873 network analyzer and the results are presented in Fig. 13. This measurement revealed that the antenna exhibits a return loss better than $10 \mathrm{~dB}$ over the frequency range from about 13 to $13.3 \mathrm{GHz}$, with a center fre- 


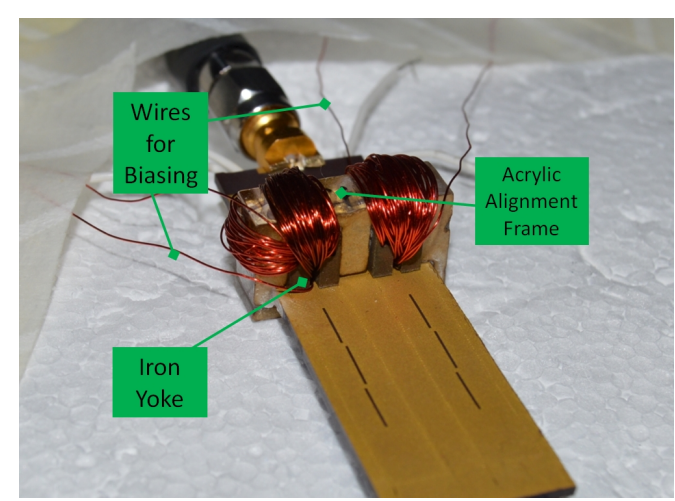

Fig. 15: Prototype under characterization with biasing circuitry.

quency of about $13.15 \mathrm{GHz}$. This measured center-frequency is slightly higher than the intended $13 \mathrm{GHz}$ predicted by simulation. It is suspected that this deviation is due to using a higher permittivity value than the actual one of the material. A post measurement simulation has been carried out by varying the permittivity within the material tolerance range $\left(\epsilon_{r}=14.6 \pm 0.6\right)$, good match was obtained by using a permittivity of 14.0 as can be seen in Fig. 13 .

The antenna radiation performance has also been characterized using SATIMO Star-Lab system. The antenna shows a maximum measured gain of $4.9 \mathrm{dBi}$ which is very close to the simulated one of $5.1 \mathrm{dBi}$ at $13.2 \mathrm{GHz}$ at no-bias. The simulated and measured $\mathrm{E}(\mathrm{xy})$ and $\mathrm{H}(\mathrm{yz})$ plane patterns are displayed in Fig. 14. As it is clear from the figure, the measured radiation pattern are in good agreement with the simulated one.

\section{B. Measurements in Biased States}

In order to test the array using the external yoke systems, we fabricated an acrylic alignment-sleeve to keep the yokes in position over the phase shifters as shown in Fig. 15. About 80 turns were wound on each yoke. Using this configuration, the beam could be steered up to \pm 28 degrees in E-plane by incrementing the current to maximum value of $1.75 \mathrm{~A}$. The measured patterns are shown in Fig. 16(a). Some distortion of the pattern could be observed (relative to the unbiased case) and is likely to be because of the wiring and bias circuitry placed around the antenna. Also, some asymmetry is observed when scanning to positive and negative angles in the external windings case. This can be caused by a misalignment error in the placement of the external coils. A gain drop of about $1 \mathrm{~dB}$ occurs when scanned to 28 degrees. Increasing the bias current beyond $1.75 \mathrm{~A}$ was not possible as it caused melting of the insulation of the copper wires and short circuiting the turns. Also, it is worth mentioning that the input impedance of the array showed very little variation when the phase shifters are biased. The measurements results of the array input reflection coefficient when one phase shifter is being biased are displayed in Fig.17. As can be seen little variation can be observed and the input of the array remains satisfactorily matched to $50 \mathrm{Ohm}$ over the frequency from about $12.98 \mathrm{GHz}$ to $13.38 \mathrm{GHz}$.

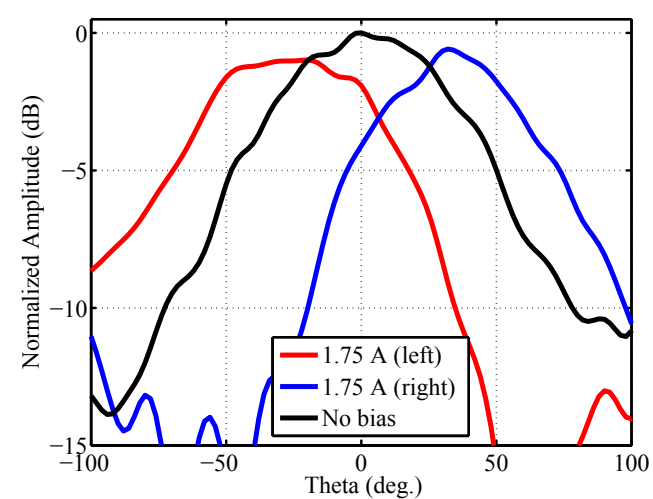

(a)

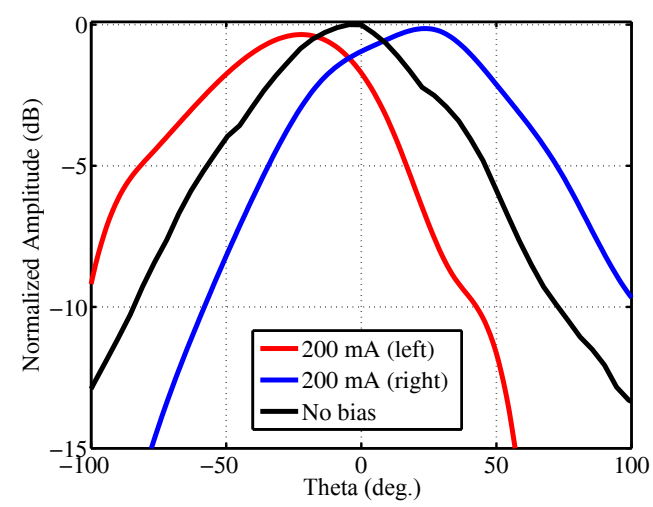

(b)

Fig. 16: Measured E-plane pattern at maximum scanning using (a) external windings and $\mathrm{I}=1.75 \mathrm{~A}$ (b) embedded windings and $\mathrm{I}=0.2 \mathrm{~A}$.

By using the embedded windings to bias the phase shifter, a maximum beam scanning of nearly \pm 19 degrees could be obtained at a $200 \mathrm{~mA}$ of applied current as depicted in Fig. 16(b). It is worth noting that the embedded windings resistance was much higher than the external one (16 ohm compared to $0.5 \mathrm{ohm}$ for the external coil). This high resistance limited the application of higher currents for biasing. At higher currents, the windings started to heat up appreciably over the course of the radiation pattern measurements resulting in a distorted pattern.

A summary of scanning results for both internal and external biasing techniques is given in table I. From the table, it can be observed that the embedded windings biasing approach can be used to magnetize the structure to a higher level for a given current compared to the external biasing approach. This is evident by observing that the embedded windings achieve about 20 degrees of scanning at $200 \mathrm{~mA}$ whereas the external windings achieve this value at $850 \mathrm{~mA}$. Compared to simulations, it is noticed that measured scanning angles for a given current are generally higher. It is believed that this deviation from simulation predictions are coming from underestimating the generated magnetization from each current value. This underestimation can be due to inaccuracy of the BH-curve used for modeling the material and/or an inaccuracy in estimating the air-gap size in case of external windings. All inclusive, the simulations predictions are found to be fairly close to actual 


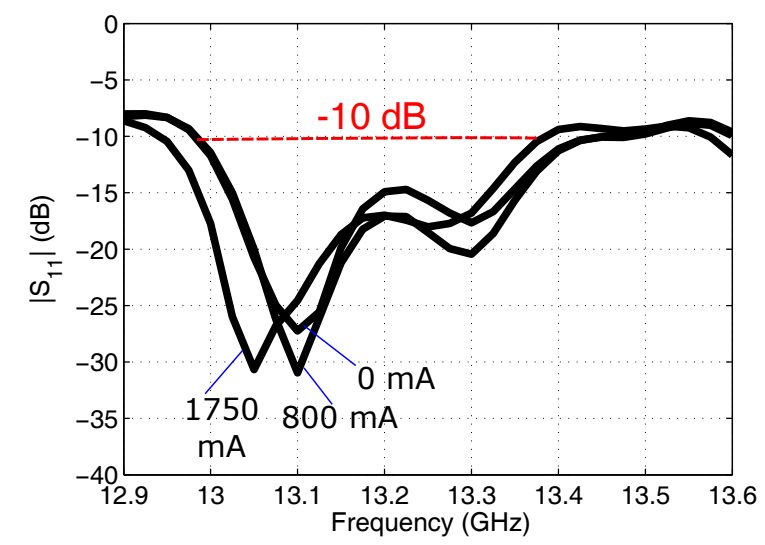

Fig. 17: Measured reflection coefficient under different bias currents.

TABLE I: Summary of results for internal and external biasing.

\begin{tabular}{|c|c|c|c|c|c|c|}
\hline \multirow{2}{*}[\mathrm{A}]{} & \multicolumn{2}{|c|}{ Embedded Windings } & \multicolumn{3}{|c|}{ External Windings } \\
\cline { 2 - 7 } & $\begin{array}{c}\text { Sim. } \\
\mathrm{M} \\
\left(M / M_{s}\right)\end{array}$ & $\Theta_{\text {sim }}$ & $\Theta_{\text {mes }}$ & $\begin{array}{c}\text { Sim. } \\
\mathrm{M} \\
\left(M / M_{s}\right)\end{array}$ & $\Theta_{\text {sim }}$ & $\Theta_{\text {mes }}$ \\
\hline 0.2 & $\begin{array}{c}1082 \mathrm{G} \\
(27 \%)\end{array}$ & 14.2 & 19 & $\begin{array}{c}541 \mathrm{G} \\
(14 \%)\end{array}$ & 7.2 & - \\
\hline 0.85 & $\begin{array}{c}2890 \mathrm{G} \\
(72 \%)\end{array}$ & 30.2 & - & $\begin{array}{c}1527 \mathrm{G} \\
(38 \%)\end{array}$ & 18.7 & 20 \\
\hline 1.75 & $\begin{array}{c}4000 \mathrm{G} \\
(100 \%)\end{array}$ & 45 & - & $\begin{array}{c}1716 \mathrm{G} \\
(43 \%)\end{array}$ & 21 & 28 \\
\hline
\end{tabular}

measurements and the deviations have small impact on the overall performance of either case.

\section{Future Ferrite LTCC Phased Arrays}

The high permittivity and moderate loss tangent of Ferrite LTCC $\left(\epsilon_{r}=14.6, \tan \delta=0.005\right)$ limits the antenna gain and efficiency. To improve the antenna performance, it is suggested that conventional low-loss LTCC material such as Dupont $9 \mathrm{k} 7\left(\epsilon_{r}=7.1, \tan \delta=0.0009\right)$ be used for antennas realization, while the phase shifters can still be implemented in the ferrite multilayer technology. This will improve the efficiency of the antenna considerably while maintaining the beam steering capability of the phased antenna array. However, this requires further research work on the fabrication side to enable monolithic integration of conventional and ferrite LTCC tape systems into a single package.

Another performance limitation for Ferrite LTCC based phased arrays comes from the self-heating of the embedded bias windings. This heating sets a limit on the allowable bias current and hence the realizable phase shift and scanning range. To mitigate this effect, the embedded windings need to be designed for minimum resistance while meeting the desired magnetization level. Additionally, in-package cooling mechanisms to dissipate heat generated from internal bias windings must be considered in future designs. One suggested way is to incorporate microfluidic channels within the LTCC package through continuous embedded cavities. Through these channels a liquid coolant can be pumped to diffuse the generated heat. With the internal windings cooled, more current can be applied resulting in higher phase shift and larger scanning range for a phased array.

In addition to cooling mechanism, improving the remanent magnetization of the ferrite material can also help reduce the generated heat as well as the consumed power. If the material has sufficiently high remanent-to-saturated magnetization ratio $\left(M_{r} / M_{s}\right)$, the bias current need not be continuously applied to maintain the material magnetized. Instead, the current need only be pulsed for a short duration (typically few hundred microseconds) to switch the magnetization level inside the material [22], [26]. This mode of operation is usually referred to as "latching" and is feasible to use when the material has near-square hysteresis $\left(M_{r} / M_{s}>0.75\right)$. Near-square hysteresis microwave ferrites are commercially available in bulk substrate form [27]. The development of similar LTCC process-compatible versions of those, currently being researched [28], can resolve self-heating problems as well as greatly reduce device power consumption.

\section{CONCLUSion}

In this work, a new implementation of SIW-based phased antenna arrays is proposed. Unlike previously demonstrated SIW phased arrays that use a SIW fixed beam-forming network (ex: butter matrix) or mountable switching elements on the SIW to switch the beam, the phase shifting is implemented by using tunable ferrite-filled SIW phase shifters. The proposed approach has the advantages of allowing for continuous beam scanning, avoiding the use of surface mount components and providing monolithic integration of the antennas with phase shifters in SIW technology. A demonstrator twoelement slotted-SIW phased antenna array prototype has been designed fabricated and measured. For testing the array, two approaches were used for providing the biasing magnetic field for the phase shifters. The first approach relies on coil systems implemented using circuitry outside the Ferrite LTCC package and the second approach used windings that are realized within the package. A maximum beam steering of \pm 28 degrees could be measured using the external biasing approach and a winding current of $1.75 \mathrm{~A}$, while a maximum scanning of about \pm 19 degrees is measured using the internal bias circuitry and a current of $0.2 \mathrm{~A}$. It is important to mention that although the internal winding is found to be more efficient in magnetizing the substrate with low currents, they generally suffer from lager windings resistance (nearly 20 times higher) than external windings of similar size. Consequently, greater power dissipation is observed in the case of the internal windings for the same current. Also, the large embedded winding resistance limits the amount of current that can be used without causing permanent damage to the conductive traces and it makes the substrate subject to self-heating effects. If embedded windings are to be used for biasing in future works, it is recommended to attempt to minimize the winding resistance as much as possible and incorporate heat dissipation techniques into the package. As a first attempt, the demonstrated prototype results show promise for using Ferrite LTCC as a platform for SIWbased phased antenna arrays. 


\section{ACKNOWLEDGMENT}

The authors would like to thank Kari Kautio and his team at VTT-electronics Finland for the efforts in fabricating the prototype and King Abdullah University of Science and Technology (KAUST) for funding this project.

\section{REFERENCES}

[1] K. W. P. A. M. Bozzi, L. Perregrini, "Current and Future Research Trends in Substrate Integrated Waveguide Technology," Radioengineering, vol. 18, no. 2, pp. 201-209, 2009.

[2] Y. J. Cheng, W. Hong, K. Wu, Z. Q. Kuai, C. Yu, J. X. Chen, J. Zhou, and H. J. Tang, "Substrate Integrated Waveguide (SIW) Rotman Lens and Its Ka-Band Multibeam Array Antenna Applications," IEEE Trans. Antennas, vol. 56, no. 8, pp. 2504-2513, Aug 2008

[3] A. Guntupalli and K. Wu, "Phase-Steered Fixed Beams in One and Two-Dimensional Scan Space for Substrate-Integrated Radar and Radio Systems at $60 \mathrm{GHz}$," IET Microwaves, Antennas and Propagation, vol. 8 , no. 11, pp. 819-828, August 2014.

[4] C.-C. Chang, R.-H. Lee, and T.-Y. Shih, "Design of a Beam Switching/Steering Butler Matrix for Phased Array System," IEEE Trans. Antennas, vol. 58, no. 2, pp. 367-374, Feb 2010.

[5] L. Wu, A. Farrall, and P. Young, "Substrate Integrated Waveguide Switched Beam Antenna," IEEE Trans. Antennas, vol. 63, no. 5, pp. 2301-2305, May 2015.

[6] P. Loghmannia, M. Kamyab, M. Ranjbar Nikkhah, and R. Rezaiesarlak, "Miniaturized Low-Cost Phased-Array Antenna Using SIW Slot Elements," IEEE Antennas Wireless Propag. Lett., vol. 11, pp. 1434-1437, 2012.

[7] X. Yang, J. Wu, Y. Gao, T. Nan, Z. Zhou, S. Beguhn, M. Liu, and N. Sun, "Compact and low loss phase shifter with low bias field using partially magnetized ferrite," IEEE Trans. Magn., vol. 49, no. 7, pp. 3882-3885, July 2013.

[8] W. Hui, J. Bell, M. Iskander, and J. Lee, "Low-Cost MicrostripLine-Based Ferrite Phase Shifter Design for Phased Array Antenna Applications," IEEE Antennas Wireless Propag. Lett., vol. 6, pp. 8689, 2007.

[9] H. Kishimoto, T. Ueda, and Y. Kado, "Experimental demonstration of nonreciprocal phase-shift composite right/left-handed transmission lines using a ferrite-rod-embedded substrate," IEEE Trans. Magn., vol. 47, no. 10, pp. 3724-3727, Oct 2011.

[10] L. Carignan, T. Kodera, D. Menard, and C. Caloz, "Moldable polymer/ferrite composite and application to an integrated cpw tunable phase shifter," IEEE Microw. Wireless Compon. Lett., vol. 19, no. 4, pp. 206208, April 2009.

[11] A. Abuelma'atti, J. Zafar, I. Khairuddin, A. Gibson, A. Haigh, and I. Morgan, "Variable toroidal ferrite phase shifter," Microwaves, Antennas Propagation, IET, vol. 3, no. 2, pp. 242-249, March 2009.

[12] W. Che, E.-N. Yung, and K. Wu, "Millimeter-wave ferrite phase shifter in substrate integrated waveguide (siw)," in Antennas and Propagation Society International Symposium, 2003. IEEE, vol. 4, June 2003, pp. $887-890$ vol.4.

[13] S. Adhikari, A. Ghiotto, S. Hemour, and K. Wu, "Tunable non-reciprocal ferrite loaded siw phase shifter," in Microwave Symposium Digest (IMS), 2013 IEEE MTT-S International, June 2013, pp. 1-3.

[14] A. Ghiotto, S. Adhikari, and K. Wu, "Ferrite-loaded substrate integrated waveguide switch," Microwave and Wireless Components Letters, IEEE, vol. 22, no. 3, pp. 120-122, March 2012.

[15] F. Ghaffar, J. Bray, and A. Shamim, "Theory and Design of a Tunable Antenna on a Partially Magnetized Ferrite LTCC Substrate," IEEE Trans. Antennas, vol. 62, no. 3, pp. 1238-1245, March 2014.

[16] F. Abdul Ghaffar and A. Shamim, "A Partially Magnetized Ferrite LTCC Based SIW Phase Shifter for Phased Array Applications," IEEE Trans. Magn., vol. PP, no. 99, pp. 1-1, 2015.

[17] A. Shamim, J. Bray, N. Hojjat, and L. Roy, "Ferrite LTCC-Based Antennas for Tunable SoP Applications," IEEE Trans. Compon. Packag. Manuf. Technol., vol. 1, no. 7, pp. 999-1006, 2011.

[18] E. Arabi, F. Ghaffar, and A. Shamim, "Tunable Bandpass Filter Based on Partially Magnetized Ferrite LTCC With Embedded Windings for SoP Applications," IEEE Microw. Compon. Lett., vol. 25, no. 1, pp. 16-18, Jan 2015.

[19] A. Nafe and A. Shamim, "An integrable siw phase shifter in a partially magnetized ferrite ltcc package," IEEE Transactions on Microwave Theory and Techniques, vol. 63, no. 7, pp. 2264-2274, July 2015.
[20] Electroscience thick-film materials and ceramic tapes. [Online]. Available: http://www.electroscience.com/pdf/40012.pdf

[21] J. J. Green and F. Sandy, "Microwave Characterization of Partially Magnetized Ferrites," IEEE Trans. Microw. Theory Techn., vol. 22, no. 6, pp. $641-645,1974$

[22] S. K. K. . B. Bhat, Microwave and Millimeter Wave Phase Shifters. Dedham, Massachusetts: Artech House Inc, 1991, vol. I, pp. 7-8, 115$116,185$.

[23] J. Bray and L. Roy, "Development of a Millimeter-Wave FerriteFilled Antisymmetrically Biased Rectangular Waveguide Phase Shifter Embedded in Low-Temperature Cofired Ceramic," IEEE Trans. Microw. Theory Techn., vol. 52, no. 7, pp. 1732-1739, 2004.

[24] R. C. Johnson, Antenna Engineering Handbook, 3rd ed. McGraw-Hill Professional, 1992.

[25] J. Massen, M. Frei, W. Menzel, and U. Möller, "A $79 \mathrm{GHz} \mathrm{SiGe}$ Short-Range Radar Sensor for Automotive Applications," International Journal of Microwave and Wireless Technologies, vol. 5, pp. 5-14, 2 2013.

[26] V. G. Harris, "Modern microwave ferrites," IEEE Trans. Magn., vol. 48, no. 3, pp. 1075-1104, 2012.

[27] Trans-tech, inc. [Online]. Available: http://www.trans-techinc.com/ products.asp

[28] T. Zhou, H. Zhang, C. Liu, L. Jin, F. Xu, Y. Liao, N. Jia, Y. Wang, G. Gan, H. Su, and L. Jia, " " $\mathrm{Li}_{2} \mathrm{O}-\mathrm{B}_{2} \mathrm{O}_{3}-\mathrm{SiO}_{2}-\mathrm{CaO}-\mathrm{Al}_{2} \mathrm{O}_{3}$ and $\mathrm{Bi}_{2} \mathrm{O}_{3}$ co-doped gyromagnetic $\mathrm{Li}_{0.43} \mathrm{Zn}_{0.27} \mathrm{Ti}_{0.13} \mathrm{Fe}_{2.1704}$ ferrite ceramics for LTCC Technology"," Ceramics International, vol. 42, no. 14, pp. 16198 $-16204,2016$

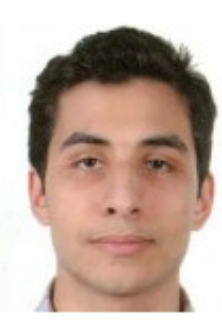

Ahmed Nafe (M'13) was born in Cairo, Egypt in 1991. He received the BSc degree in electrical engineering with highest honors from the German University in Cairo (GUC) in 2012, and the MSc degree in electrical engineering from King Abdullah University of Science and Technology (KAUST), Saudi Arabia in 2014.

$\mathrm{He}$ was a DAAD-sponsored exchange student at ULM University, Germany in 2011 where he did his BSc thesis on design and characterization of millimeter-wave antenna arrays for automotive radars. In the summer of 2011, he joined the Mixed Signal CMOS Circuits group at RWTH Aachen as a research assistant working on power amplifier linearization through digital pre-distortion in a multilevel LINC transmitter for WCDMA and LTE signals.

Mr. Nafe is a recipient of the GUC scholarship for engineering study in 2008, KAUST graduate student fellowship in 2012, and IEEE Magnetics Society Summer School sponsorship in 2015. His current research interests include analysis and design of reciprocal and non-reciprocal microwave ferrite-based components, microwave and millimeter-wave antennas and arrays, substrate integrated waveguides and power amplifier linearization techniques.

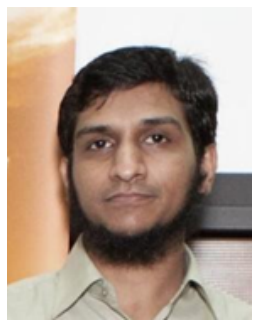

Farhan A. Ghaffar received his B.E. degree in electronics engineering from NED University of Engineering and Technology, Karachi, Pakistan in 2007 and the M.S degree in electrical engineering from King Abdullah University of Science and Technology (KAUST), Thuwal, Saudi Arabia in 2010. He is currently a $\mathrm{PhD}$ candidate in electrical engineering program at King Abdullah University of Science and Technology

Before joining KAUST Mr. Ghaffar was an Assistant Manager at Pakistan Space and Upper Atmosphere Research Commission (SUPARCO) from January 2008 to Augus 2009. He was a visiting researcher at Carleton University, Canada and Royal Military College, Canada in 2010 and 2012 respectively. His research interests involve design of System on Package (SoP) and System on Chip (SoC) based antennas, radio frequency integrated circuits (RFICs) and ferrite Low Temperature Co-fired Ceramic (LTCC) based tunable antennas and passives. 


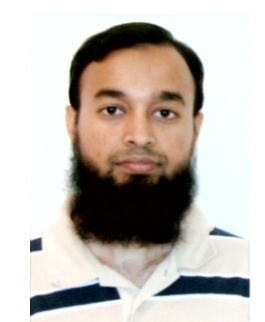

Muhammad Fahad Farooqui received his B.E. degree in electronics engineering from NED University of Engineering and Technology, Karachi, Pakistan and the M.S degree in electrical engineering from Texas A\&M university, USA. He is currently a $\mathrm{PhD}$ candidate in electrical engineering program at King Abdullah University of Science and Technology. His research interests include wireless sensors for internet of things (IoT), inkjet-printed and 3Dprinted radio frequency (RF) electronics, antennas and $\mathrm{RF} /$ microwave circuits. During his graduate studies, Fahad has authored more than 15 international publications which include 7 peer reviewed journal papers.

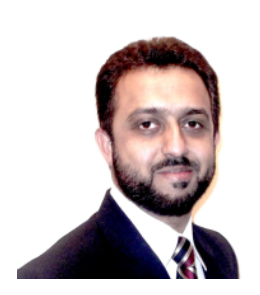

(Oulu, Finland) in 2006. Dr. Shamim was the recipient of the best paper prize at the European Microwave Association Conference in 2008. He was given the Ottawa Centre of Research Innovation (OCRI) Researcher of the Year 2008 Award in Canada. His work on Wireless Dosimeter won the ITAC SMC Award at Canadian Microelectronics Corporation TEXPO in 2007. He received the best student paper finalist prize at IEEE APS conference in 2005. He also won numerous business related awards, including 1st prize in Canada's national business plan competition and was selected for OCRI Entrepreneur of the year award in 2010. He is an author/co-author of over 80 international publications and an inventor on 9 patents. His research interests are in integrated on-chip antennas, low power CMOS RFICs for system-on-chip (SoC) applications and advanced system-on-package (SoP) designs in multilayer LTCC, LCP, and paper substrates through screen and inkjet printing techniques. Dr. Shamim is a Senior Member of IEEE and serves on the editorial board of IEEE Transactions on Antennas and Propagation. He is the founding chair of IEEE MTTS and APS joint chapter for Western Saudi Arabia. 